\title{
A STRICT INCLUSION RELATED TO BIHARMONIC GREEN'S FUNCTIONS OF CLAMPED AND SIMPLY SUPPORTED BODIES
}

\author{
MITSURU NAKAI and LEO SARIO
}

Let $O_{\beta}^{N}$ and $O_{\gamma}^{N}$ be the classes of Riemannian $N$-manifolds, $N \geqq 2$, which do not carry biharmonic Green's functions $\beta$ of clamped bodies, characterized by "boundary data" $\beta=\partial \beta / \partial n=0$, or biharmonic Green's functions $\gamma$ of simply supported bodies, characterized by boundary data $\gamma=\Delta \gamma=0$, respectively. It is known that $O_{\beta}^{N} \subset O_{\gamma}^{N}$ (Ralston-Sario [4]), but whether or not this inclusion is strict has been an open problem. The main purpose of the present study is to show that the inclusion is strict:

$$
O_{\beta}^{N}<O_{\gamma}^{N} .
$$

Let $O_{G}^{N}$ be the class of parabolic Riemannian manifolds, i.e., those not carrying harmonic Green's functions. For any null class $O^{N}$, denote by $\widetilde{O}^{N}$ its complement. It is known that $O_{G}^{N}<O_{\gamma}^{N}$ (Sario [5]), but the relation of $O_{G}^{N}$ to $O_{\beta}^{N}$ has been unknown, except for the special case $N=2$, in which the invariance of harmonicity under conformal metrics allowed us to construct 2-manifolds which belong to $O_{G}^{2} \cap \widetilde{O}_{\beta}^{2}$, (Nakai-Sario [3]). We shall now show that, for any $N>2$ as well, there exist $N$-manifolds which are parabolic but nevertheless carry $\beta$ :

$$
O_{G}^{N} \cap \widetilde{O}_{\beta}^{N} \neq \emptyset \text {. }
$$

This relation is sharper than $O_{\beta}^{N}<O_{\gamma}^{N}$.

A perhaps somewhat unexpected consequence of our reasoning will be that, for $N=2,3$, every compact Riemannian manifold punctured at a point carries $\beta$.

We start by giving, in No. 1 , a new short proof of the Ralston-Sario relation $O_{\beta}^{N} \subset O_{\gamma}^{N}$. In No. 2, we introduce a useful sufficient condition for the existence of $\beta$ on parabolic manifolds: an Evans kernel is square integrable off its pole. We use this test to show, in No. 3, that the parabolic Riemannian ball constructed in NakaiSario [2] actually carries $\beta$.

This work was supported by Grant DA-ARO-31-124-73-G39, University of California, Los Angeles.

MOS Classification 31 B 30.

doi:10.5186/aasfm.1977.0319 
L. Chung has reported to the authors that he has constructed another counterexample to show the strictness of $O_{\beta}^{N}<O_{\gamma}^{N}$. His manifold is the $N$-space with a nonconformal metric.

1. New proof of $O_{\beta}^{N} \subset O_{\gamma}^{N}$. To show that $O_{\beta}^{N} \subset O_{\gamma}^{N}$, take a manifold $R$ in $\widetilde{O}_{\gamma}^{N}$, $N \geqq 2$. Choose a regular subregion $\Omega$ of $R$ and denote by $g_{\Omega}(x, y)$ the harmonic Green's function in $\bar{\Omega}$. Let $\beta_{\Omega}(x, y)$ be the biharmonic Green's function of the clamped body on $\bar{\Omega}$, characterized by $\Delta^{2} \beta_{\Omega}=\Delta \Delta \beta_{\Omega}=u$ on $\Omega-\{y\}$, the biharmonic fundamental singularity at $y$, and the conditions $\beta_{\Omega}=\partial \beta_{\Omega} / \partial n=0$ on $\partial \Omega$. Here $\Delta$ is the Laplace-Beltrami operator $d \delta+\delta d$. Write

$$
s_{\Omega}(x, y)=\Delta_{x} \beta_{\Omega}(x, y)
$$

and set $s_{\Omega}(\cdot, y)=g_{\Omega}(\cdot, y)=0$ on $R-\bar{\Omega}$. Then

$$
\beta_{\Omega}(x, y)=\left(g_{\Omega}(x, \cdot), s_{\Omega}(\cdot, y)\right) .
$$

Let $h \in H(\Omega) \cap C^{1}(\bar{\Omega})$, where $H$ stands for the class of harmonic functions. In view of

and

$$
\int_{\partial \Omega} h(x) *_{x} d \beta_{\Omega}(x, y)=0
$$

we have

$$
\left(d h, d \beta_{\Omega}\right)_{\Omega}=\int_{\partial \Omega} \beta_{\Omega} * d h=0
$$

for all $h \in H(\Omega) \cap C^{1}(\bar{\Omega})$.

$$
\left(h(\cdot), s_{\Omega}(\cdot, y)\right)_{\Omega}=0
$$

Fix $x, y \in R$ and take regular subregions $\Omega_{0}, \Omega_{1}$ with $\bar{\Omega}_{0} \subset \Omega_{1}$ and $x, y \in \Omega_{0}$. By Harnack's inequality, the existence of the biharmonic Green's function of a simply supported body on $R$,

is equivalent to

$$
\gamma_{R}(x, y)=\left(g_{R}(\cdot, x), g_{R}(\cdot, y)\right)
$$

$$
\left\|g_{R}(\cdot, x)\right\|_{R-\Omega_{1}}<\infty
$$

for every $x \in \Omega_{0}$. Since $\beta_{\Omega}(x, y)=\left(g_{\Omega}(\cdot, x), s_{\Omega}(\cdot, y)\right)_{\Omega}$, we obtain

$$
\beta_{\Omega^{\prime}}(x, y)-\beta_{\Omega}(x, y)=\left(g_{R}(\cdot, x)-s_{\Omega_{1}}(\cdot, x), s_{\Omega^{\prime}}(\cdot, y)-s_{\Omega}(\cdot, y)\right)_{\Omega^{\prime}}
$$

for $\Omega \subset \Omega^{\prime}$ with $\Omega_{1} \subset \Omega$ and for any $x \in \Omega_{0}$. The quantity

$$
K=\sup _{x \in \Omega_{0}}\left\|g_{R}(\cdot, x)-s_{\Omega_{1}}(\cdot, x)\right\|_{R}
$$

is finite by virtue of the continuity of $g_{R}(z, x)-s_{\Omega_{1}}(z, x)$ on $\Omega_{1} \times \Omega_{1}$. The Schwarz inequality yields

$$
\left|\beta_{\Omega^{\prime}}(x, y)-\beta_{\Omega}(x, y)\right|^{2} \leqq K^{2}\left\|s_{\Omega^{\prime}}(\cdot, y)-s_{\Omega}(\cdot, y)\right\|_{\Omega^{\prime}}^{2}
$$

for $\Omega^{\prime} \supset \Omega \supset \Omega_{1}$ and $x \in \Omega_{0}$. Here,

$$
\left\|s_{\Omega^{\prime}}(\cdot, y)-s_{\Omega}(\cdot, y)\right\|_{\Omega^{\prime}}^{2}=\left\|s_{\Omega^{\prime}}(\cdot, y)-s_{\Omega_{1}}(\cdot, y)\right\|_{\Omega^{\prime}}^{2}-\left\|s_{\Omega}(\cdot, y)-s_{\Omega_{1}}(\cdot, y)\right\|_{\Omega^{2}}^{2} .
$$


Since

we obtain

$$
\left(g_{R}(\cdot, y)-s_{\Omega}(\cdot, y), s_{\Omega}(\cdot, y)-s_{\Omega_{1}}(\cdot, y)\right)_{\Omega}=0
$$

$$
\left(g_{R}(\cdot, y)-s_{\Omega_{1}}(\cdot, y), s_{\Omega}(\cdot, y)-s_{\Omega_{1}}(\cdot, y)\right)_{\Omega}=\left\|s_{\Omega}(\cdot, y)-s_{\Omega_{1}}(\cdot, y)\right\|_{\Omega}^{2} .
$$

The Schwarz inequality gives

$$
\left\|s_{\Omega}(\cdot, y)-s_{\Omega_{1}}(\cdot, y)\right\|_{\Omega} \leqq\left\|g_{R}(\cdot, y)-s_{\Omega_{1}}(\cdot, y)\right\|_{R} \leqq K
$$

for every $\Omega$. Therefore,

and

$$
\lim _{\Omega^{\prime} \supset \Omega \nearrow R}\left\|s_{\Omega^{\prime}}(\cdot, y)-s_{\Omega}(\cdot, y)\right\|_{\Omega^{\prime}}^{2}=0
$$

$$
\lim _{\Omega^{\prime} \supset \Omega \rtimes R}\left|\beta_{\Omega^{\prime}}(x, y)-\beta_{\Omega}(x, y)\right|=0,
$$

uniformly for $x \in \Omega_{0}$. Thus,

$$
\beta_{R}(x, y)=\lim _{\Omega \nearrow R} \beta_{\Omega}(x, y)
$$

exists on $R$ for any fixed $y$, and the convergence is uniform for $x$ in any compact subset of $R$.

The proof of $O_{\beta}^{N} \subset O_{\gamma}^{N}$ is complete.

2. A criterion for the existence of $\beta$. Suppose $R \in O_{G}^{N}$, and let $e(x, y)$ be an Evans kernel in the sense of Nakai [1]. For the definition and properties of $e(x, y)$ to be used below, we refer to Sario-Nakai [6, pp. 353-361]; the discussion there is for Riemann surfaces, but it applies verbatim to Riemannian manifolds. Let $B_{y}$ be a geodesic ball $|x-y|<\varepsilon$ about $y$.

Theorem 1. If an Evans kernel e on $R \in O_{G}^{N}$ satisfies

for every $y$, then $R \in \widetilde{O}_{\beta}^{N}$.

$$
\|e(\cdot, y)\|_{R-B_{y}}<\infty
$$

Proof. Using $h(\cdot)=e(\cdot, y)-s_{\Omega}(\cdot, y)$, we have, by the convention $s_{\Omega^{\prime}}(\cdot, y)=0$ on $R-\bar{\Omega}^{\prime}$,

$$
\left(e(\cdot, y)-s_{\Omega}(\cdot, y), s_{\Omega^{\prime}}(\cdot, y)\right)_{\Omega}=0
$$

for $\Omega^{\prime}=\Omega \supset \bar{B}_{y}$ and $\Omega^{\prime}=B_{y}$. We set $f(\cdot)=e(\cdot, y)-S_{B_{y}}(\cdot, y)$ and $t_{\Omega}(\cdot)=$ $=s_{\Omega}(\cdot, y)-s_{B_{y}}(\cdot, y)$ and obtain

By the Schwarz inequality,

$$
\left(f(\cdot)-t_{\Omega}(\cdot), t_{\Omega}(\cdot)\right)_{\Omega}=0
$$

$$
\left\|t_{\Omega}(\cdot)\right\|_{\Omega}^{2}=\left(f(\cdot), t_{\Omega}(\cdot)\right)_{\Omega} \leqq\|f(\cdot)\|_{\Omega} \cdot\left\|t_{\Omega}(\cdot)\right\|_{\Omega} \cdot
$$

In view of the assumption of the theorem, and the joint continuity of $e(x, y)$ on $R \times R$,

$$
\left\|s_{\Omega}(\cdot, y)-s_{B_{y}}(\cdot ; y)\right\|_{\Omega}^{2} \leqq\left\|e(\cdot, y)-s_{B_{y}}(\cdot, y)\right\|_{R}^{2}=K(y)<K\left(R_{0}\right)<\infty
$$


for every $\Omega$ and for all $y$ in an arbitrarily chosen compact subset $R_{0}$ of $R$. We recall that

$$
\begin{gathered}
\beta_{\Omega}(x, y)=\left(s_{\Omega}(\cdot, x), s_{\Omega}(\cdot, y)\right)_{\Omega} \\
\beta_{\Omega_{0}}(x, y)=\left(s_{\Omega}(\cdot, x), s_{\Omega_{0}}(\cdot, y)\right)_{\Omega},
\end{gathered}
$$

where we again use the convention $s_{\Omega^{\prime}}(\cdot, x)=0$ on $R-\bar{\Omega}^{\prime}$ for every $\Omega^{\prime}=\Omega$, $\Omega_{0} \subset \Omega$. It follows that

By the Schwarz inequality,

$$
\begin{aligned}
\beta_{\Omega}(x, y)-\beta_{\Omega_{0}}(x, y) & =\left(s_{\Omega}(\cdot, x), s_{\Omega}(\cdot, y)-s_{\Omega_{0}}(\cdot, y)\right)_{\Omega} \\
& =\left(s_{\Omega}(\cdot, x)-s_{\Omega_{0}}(\cdot, x), s_{\Omega}(\cdot, y)-s_{\Omega_{0}}(\cdot, y)\right)_{\Omega} .
\end{aligned}
$$

$$
\begin{aligned}
\left|\beta_{\Omega}(x, y)-\beta_{\Omega_{0}}(x, y)\right|^{2} & \leqq\left\|s_{\Omega}(\cdot, x)-s_{\Omega_{0}}(\cdot, x)\right\|_{\Omega}^{2} \cdot\left\|s_{\Omega}(\cdot, y)-s_{\Omega_{0}}(\cdot, y)\right\|_{\Omega}^{2} \\
& =I_{1}(x)^{2} \cdot I_{2}(y)^{2},
\end{aligned}
$$

where

$$
\begin{aligned}
I_{1}(x) & =\left\|s_{\Omega}(\cdot, x)-s_{\Omega_{0}}(\cdot, x)\right\|_{\Omega} \\
& \leqq\left\|s_{\Omega}(\cdot, x)-s_{B_{x}}(\cdot, x)\right\|_{\Omega}+\left\|s_{\Omega_{0}}(\cdot, x)-s_{B_{x}}(\cdot, x)\right\|_{\Omega} \\
& \leqq 2 K\left(R_{0}\right)^{1 / 2}<=0
\end{aligned}
$$

for all $x \in R_{0}$. Since

for all $\Omega$,

$$
\left\|s_{\Omega}(\cdot, y)-s_{B_{y}}(\cdot, y)\right\| \leqq K(y)^{1 / 2}<\infty
$$

$$
I_{2}(y)=\left\|\left(s_{\Omega}(\cdot, y)-s_{B_{y}}(\cdot, y)\right)-\left(s_{\Omega_{0}}(\cdot, y)-s_{B_{y}}(\cdot, y)\right)\right\|_{\Omega} \rightarrow 0
$$

as $\Omega \supset \Omega_{0} \nearrow R$. We conclude that

$$
\beta(x, y)=\lim _{\Omega \rightarrow R} \beta_{\Omega}(x, y)=\lim _{\Omega \rightarrow R} \beta_{\Omega}(y, x)
$$

exists and the convergence is uniform on every compact subset of $R$ for any fixed $y \in R$. The proof of Theorem 1 is complete.

We note in passing the following immediate consequence of Theorem 1:

Corollary. For $N=2,3$, every compact Riemannian manifold punctured at a point carries $\beta$.

3. Strictness of the inclusion. We are ready to establish our main result:

Theorem 2. For $N \geqq 2$,

More precisely,

$$
O_{\beta}^{N}<O_{\gamma}^{N} \text {. }
$$

$$
O_{G}^{N} \cap \widetilde{O}_{\beta}^{N} \neq \emptyset \text {. }
$$

Proof. For $N=2$, the proof was given in Nakai-Sario [3], where a necessary and sufficient condition was established for the complex plane with a conformal radial metric to carry $\beta$. For $N>2$, consider the $N$-ball

$$
R=\{r<1, d s\}
$$

with the metric $d s=\lambda(x)^{1 / 2}|d x|$, where $r=|x|, x=\left(x^{1}, \ldots, x^{N}\right), \lambda \in C^{\infty}(R), \lambda>0$, and on $\{1 / 2<r<1\}$,

$$
\lambda(x)=|x|^{(2-2 N) /(N-2)}(1-|x|)^{4 /(N-2)} .
$$


Since the function $h(x)=1 /(1-|x|)$ satisfies on $\{1 / 2<|x|<1\}$ the harmonic equation

$$
\Delta h(r)=-g^{-1 / 2}\left(g^{1 / 2} g^{r r} h^{\prime}(r)\right)^{\prime}=0,
$$

$R$ is parabolic. Therefore, there exists an Evans kernel $e(x, y)$ on $R$ such that

By virtue of

$$
e(x, y) \sim \frac{1}{1-|x|} \quad \text { as } \quad|x| \rightarrow 1 .
$$

$$
g(x)^{1 / 2}=\lambda(x)^{N / 2} \sim\left[(1-|x|)^{4 /(N-2)}\right]^{N / 2}=(1-|x|)^{2 N /(N-2)}
$$

we obtain for $\varrho \in(|y|, 1)$,

$$
\begin{aligned}
\|e(\cdot, y)\|_{|x|>e}^{2} & \sim \int_{\varrho}^{1}(1-r)^{-2}(1-r)^{2 N /(N-2)} r^{N-1} d r \\
& \sim \int_{\varrho}^{1}(1-r)^{-2+2 N /(N-2)} d r \\
& =\int_{\varrho}^{1}(1-r)^{4 /(N-2)} d r<\infty .
\end{aligned}
$$

By Theorem $1, \beta$ exists on $R$, hence $O_{G}^{N} \cap \widetilde{O}_{\beta}^{N} \neq \emptyset$.

\section{References}

[1] NaKaI, M.: On Evans' kernel. - Pacific J. Math. 22, 1967, 125-137.

[2] NakaI, M., and L. Sario: A parabolic Riemannian ball. - Proc. 1966 Amer. Math. Soc. Summer Inst., Amer. Math. Soc., Providence, 1968, 341-349.

[3] NAKaI, M., and L. SARIo: Parabolic Riemannian planes carrying biharmonic Green's functions of the clamped plate. - J. Analyse Math. 30, 1976, 372-389.

[4] Ralston, J., and L. SARIo: A relation between biharmonic Green's functions of simply supported and clamped bodies. - Nagoya Math. J. 61, 1976, 59-71.

[5] SARIO, L.: Biharmonic Green's functions and harmonic degeneracy, - J. Math. Kyoto Univ. $15,1975,351-362$.

[6] SARIO, L., and M. NAKAI: Classification theory of Riemann surfaces. - Grundlehren der Mathematischen Wissenschaften, Vol. 164, Springer-Verlag, Berlin-HeidelbergNew York, 1970.

Nagoya Institute of Technology

Department of Mathematics

Gokiso, Shôwa

Nagoya 466

Japan
University of California, Los Angeles

Department of Mathematics

Los Angeles, California 90024

USA

Received 21 September 1975 